\title{
Constraints on charged-Higgs-boson couplings
}

\author{
L. F. Abbott, P. Sikivie, and Mark B. Wise \\ Stanford Linear Accelerator Center, Stanford University, Stanford, California 94305
}

(Received 2 July 1979)

\begin{abstract}
Using the experimental value of the $K_{L}-K_{S}$ mass difference, we derive constraints on charged-Higgs-boson couplings in gauge theories of the weak and electromagnetic interactions. These bounds severely restrict the possible magnitude of charged-Higgs-boson effects in $K$ and $D$ meson decays. Our results are based on the observation that charged-Higgs-boson-exchange contributions to $K^{0}-\bar{K}^{0}$ mixing are of order $1 / M_{H}{ }^{2}$ whereas the corresponding $W$-boson contribution is only of order $m_{c}{ }^{2} / M_{W}{ }^{4}$.
\end{abstract}

\section{INTRODUCTION}

The extremely small value of the $K_{L}-K_{S}$ mass difference ${ }^{1}$ imposes severe constraints on gauge theories of the weak and electromagnetic interactions. The small $K^{0}-\bar{K}^{0}$ mixing implied by this mass difference (as well as the absence of strangeness-changing neutral-current decays) led Glashow, Iliopoulos, and Maiani ${ }^{2}$ (GIM) to introduce a fourth (charmed) quark which, when incorporated into the original Weinberg-Salam model, ${ }^{3}$ cancels unwanted strangness-changing neutral currents due to $Z^{0}$ exchange. Gaillard and $\mathrm{Lee}^{4}$ then computed the contribution to $K^{0}-\bar{K}^{0}$ mixing coming from the two- $W$-boson-exchange graph of Fig. 1 to estimate the mass of the charmed quark. In a six-quark model, the analogous calculation limits the size of the mixing angles through which the $t$ quark couples to $s$ and $d$ quarks. ${ }^{5}$ The quantumchromodynamics (QCD) corrections to the Gaillard-Lee calculation have been computed in the leading-logarithm approximation ${ }^{6,7}$ and are quite small.

It is our purpose to examine the constraints imposed on the Higgs sector of a weak and electromagnetic gauge theory by the small measured value of the $K_{L}-K_{S}$ mass difference. In the standard Weinberg-Salam model with one Higgs doublet, only a single physical scalar remains after spontaneous symmetry breakdown, the neutral Higgs particles. Since the neutral Higgs field is the same field which gives the quarks their masses (through a nonvanishing vacuum expectation value), the redefinition of quark fields which diagonalizes the quark mass matrix will also diagonalize the couplings of the neutral Higgs boson. Hence, the neutral-Higgs-boson couplings will be flavor conserving. However, in a model with more than one Higgs doublet, Higgs-bosonmediated neutral-current interactions can change flavor and so can contribute to $K^{0}-\bar{K}^{0}$ mixing. Since we seem to have at least three doublets of quarks and leptons, it is perhaps not unreason- able to consider multiple doublets in the scalar sector as well.

The first constraint imposed on models with two or more Higgs doublets by the small $K_{L}-K_{S}$ mass difference is that strangeness-changing interactions due to neutral-Higgs-boson exchange must be suppressed. This can be done by requir ing that certain Higgs-boson Yukawa couplings vanish. One can impose this "naturally" ${ }^{8}$ by invoking some kind of discrete symmetry. We will consider two such models. For simplicity we will restrict our discussion to models with two Higgs doublets. It is straightforward to extend our results to other models and to cases in which there are three or more Higgs doublets.

We have already noted that when one scalar field gives the quarks their masses, then the transformation which diagonalizes the quark mass matrix will also diagonalize the couplings of the neutral component of that scalar field. Glashow and Weinberg ${ }^{9}$ noted that this argument can be made separately for charge $\frac{2}{3}$ quarks and for charge $-\frac{1}{3}$ quarks, since by charge conservation the mass matrix can never mix these two types of quarks. They thus pointed out that strangenesschanging neutral-Higgs -boson exchange can be avoided in a model with more than one Higgs doublet if the neutral component of one scalar field is coupled to charge $\frac{2}{3}$ quarks and the neutral component of one other scalar field is coupled to the charge $-\frac{1}{3}$ quarks. The coupling of the charged components is then determined by $\mathrm{SU}(2) \otimes \mathrm{U}(1)$ symmetry. Weinberg has discussed Higgs-bosoninduced $C P$ violation in this model when three or more Higgs doublets are present. ${ }^{10}$ Since we are interested in the magnitude of Higgs-boson couplings we will, for simplicity, restrict our attention to the model with only two Higgs doublets in which case there is no $C P$ violation coming from charged-Higgs-boson propagators. (However, as usual $C P$ violation can still arise in the six-quark version of this model from a complex phase in the Kobayashi-Maskawa matrix. ${ }^{11}$ ) In any case, as 


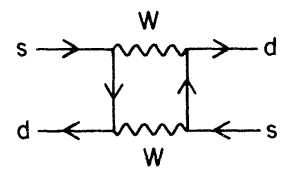

FIG. 1. The two- $W$ exchange contribution to the effective Hamiltonian for $K^{0}-\bar{K}^{0}$ mixing.

mentioned above, the extension of our results to the case discussed by Weinberg is straightforward.

In the second model which we will consider, flavor-changing neutral-Higgs-boson exchange is avoided by requiring that only one of the two Higgs doublets couples to the quarks. Since this one scalar field must give the quarks their masses, nondiagonal neutral Higgs-boson couplings are eliminated in the standard way. This model has been discussed by Haber, Kane, and Sterling. ${ }^{12}$ The two models we are considering are described in more detail in Sec. II.

Although flavor-changing neutral Higgs-boson couplings have been eliminated in these two models at the tree level, there can still be significant flavor-changing interactions from charged-Higgsboson exchange. To lowest order, these will contribute to $K^{0}-\bar{K}^{0}$ mixing through the diagrams of Figs. 2 and 3. Both of the scalar doublets in these models can have nonvanishing vacuum expectation values and we will label the magnitudes of these by $\xi$ and $\eta$. Then the charged-Higgs-boson couplings to quarks will be of order $g\left(m_{q} / M_{W}\right)(\xi / \eta)$ where $m_{q}$ is a quark mass and $M_{W}$ is the $W$-boson mass. Clearly, large values of $(\xi / \eta)$ would be required to produce significant charged-Higgs boson exchange effects for "light" $(u, d, s, c)$ quarks. There is no a priori reason why Higgs-boson couplings cannot be large in models with mult $\iota_{\text {ple }}$ Higgs doublets, and it has been shown ${ }^{12}$ that large Higgs-boson couplings are not in contradiction with data on particle decays. Naively, one would expect that $W$ and Higgs-boson exchange processes would be comparable when

$$
\frac{g^{2}}{M_{W}^{2}} \approx \frac{g^{2}}{M_{H}^{2}} \frac{m_{q}^{2}}{M_{W}^{2}}\left(\frac{\xi}{\eta}\right)^{2}
$$

or equivalently, when

$$
\left(\frac{\xi}{\eta}\right)^{2} \approx \frac{M_{H}^{2}}{m_{q}^{2}}
$$

However, in the case of $K^{0}-\bar{K}^{0}$ mixing the Higgs-

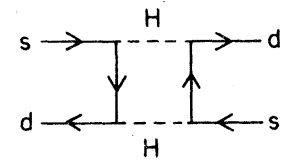

FIG. 2. The two-Higgs-boson exchange contribution to the effective Hamiltonian for $K^{0}-\bar{K}^{0}$ mixing. boson-exchange diagrams of Figs. 2 and 3 are in fact much more important relative to the two- $W$ exchange process (Fig. 1) than this simple discussion would indicate. Let us compare, for example, Figs. 1 and 2 in the four-quark model. Individual diagrams such as Fig. 1 are of order $1 / M_{W}^{2}$; however, when contributions from $c$ and $u$ quarks are added, there is a cancellation related to the GIM cancellation of flavor-changing $Z^{0}$ coupl ings and the final result for two- $W$ exchange is of order $m_{c}^{2} / M_{W}{ }^{4}$. In Fig. 2, on the other hand, this cancellation is spoiled by the presence of quark masses at the Higgs-boson vertices, so the result for two-Higgs-boson exchange is of order $(\xi / \eta)^{4}\left(m_{c} / M_{W}\right)^{4}\left(1 / M_{H}^{2}\right)$, not of order $(\xi / \eta)^{4}\left(m_{c} / M_{W}\right)^{4}\left(m_{c}^{2} / M_{H}{ }^{4}\right)$. Thus, the Higgs boson diagram is a factor $M_{H}^{2} / m_{c}^{2}$ larger than one might naively have expected. This fact enables us, in Sec. III, to derive a bound for $(\xi / \eta)^{2}$ which indicates that $(\xi / \eta)^{2}$ must be smaller than Eq. (1.2) by an order of magnitude or more for a large range of Higgs-boson masses. We should point out that a large Higgs-boson contribution to $K^{0}-\bar{K}^{0}$ mixing such as we have found is a general feature of any model in which the order $1 / M_{H}{ }^{2}$ contributions to the charged-Higgs-boson exchange graphs (Figs. 2 and 3 ) do not cancel. Thus, the small $K_{L}-K_{S}$ mass difference will impose severe constraints on the parameters of any such model.

The QCD corrections to the Higgs-boson-exchange contribution to $K^{0}-\bar{K}^{0}$ mixing, which are discussed in Sec. IV, are much larger than those for the $W$-exchange graph of Fig. 1. This is due to the different structure of the Higgs-boson vertices. Although the QCD corrections to our bound on $(\xi / \eta)^{2}$ are fairly large, their effects on its phenomenological implications are small because similar QCD corrections affect all Higgs - boson exchange processes involving quarks. Some phenomenological implications of our bound are discussed in Sec. V.

\section{MODELS WITH TWO HIGGS DOUBLETS}

We consider models with two Higgs doublets

$$
\tilde{\phi}_{1}=\left(\begin{array}{c}
\tilde{\phi}_{1}^{0} \\
\tilde{\phi}_{1}^{-}
\end{array}\right), \quad \tilde{\phi}_{2}=\left(\begin{array}{c}
\tilde{\phi}_{2}^{0} \\
\tilde{\phi}_{2}^{-}
\end{array}\right) \text {. }
$$

For a general Higgs-boson potential, the vacuum is characterized by two vacuum expectation values $^{13}$

$$
\left\langle\tilde{\phi}_{1}\right\rangle=\left(\begin{array}{l}
\eta \\
0
\end{array}\right),\left\langle\tilde{\phi}_{2}\right\rangle=e^{i \epsilon}\left(\begin{array}{l}
\xi \\
0
\end{array}\right),
$$

where $\eta, \xi$, and $\epsilon$ are real and positive. In order to identify the Goldstone bosons of these models 


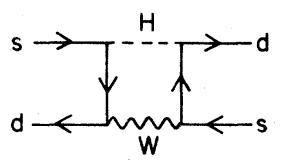

FIG. 3. The mixed Higgs-boson- $W$ exchange contribution to the effective Hamiltonian for $K^{0}-\bar{K}^{0}$ mixing. (The diagram with Higgs-boson and $W$ propagators exchanged is not considered separately as it is automatically taken into account in the effective-operator formalism.)

we define rotated fields

$$
\begin{aligned}
& \phi_{1}=\cos \alpha \tilde{\phi}_{1}+e^{-i \epsilon} \sin \alpha \tilde{\phi}_{2}, \\
& \phi_{2}=-\sin \alpha \tilde{\phi}_{1}+e^{-i \epsilon} \cos \alpha \tilde{\phi}_{2},
\end{aligned}
$$

where

$$
\begin{aligned}
& \sin \alpha=\frac{\xi}{\left(\eta^{2}+\xi^{2}\right)^{1 / 2}}, \\
& \cos \alpha=\frac{\eta}{\left(\eta^{2}+\xi^{2}\right)^{1 / 2}} .
\end{aligned}
$$

Then we find that only $\phi_{1}$ has a nonvanishing vacuum expectation value

$$
\left\langle\phi_{1}\right\rangle=\left(\begin{array}{c}
\left(\eta^{2}+\xi^{2}\right)^{1 / 2} \\
0
\end{array}\right),\left\langle\phi_{2}\right\rangle=0 .
$$

In the language of Georgi and Nanopoulos, ${ }^{14} \phi_{1}$ is the "true" Higgs field. The charged components of $\phi_{1}$ are the charged Goldstone bosons which join with the charged gauge bosons to make the massive $W^{ \pm}$. One of the neutral components of $\phi_{1}$, $\operatorname{Im}\left(\phi_{1}^{0}\right)$, is the neutral Goldstone boson which becomes part of the massive $Z^{0}$. The remaining physical scalar particles are the charged components of $\phi_{2}$ and three neutral scalars which are formed from linear combinations of $\operatorname{Re}\left(\phi_{1}^{0}\right), \operatorname{Re}\left(\phi_{2}^{0}\right)$, and $\operatorname{Im}\left(\phi_{2}^{0}\right)$. Note that the charged components of $\phi_{2}$, which are the charged Higgs particles we are interested in, are necessarily mass eigenstate ${ }^{15}$ since the only fields they could mix with $\phi_{1}^{ \pm}$are the Goldstone bosons and would not even appear in the Lagrangian in unitary gauge. $\mathrm{Be}-$ cause of this absence of mixing, the chargedHiggs-boson propagator when only two doublets are present can have no complex phase factors, and $C P$ violation will not occur through chargedHiggs-boson exchange (except for the phase factor in the Kobayashi-Maskawa matrix ${ }^{11}$ ).

Let us define quark fields which are weak-interaction eigenstates by

$$
\mathcal{u}^{\prime}=\left(\begin{array}{c}
u^{\prime} \\
c^{\prime} \\
t^{\prime}
\end{array}\right), \quad \mathbb{D}^{\prime}=\left(\begin{array}{l}
d^{\prime} \\
s^{\prime} \\
b^{\prime}
\end{array}\right) \text {. }
$$

The first model which we mentioned in the Introduction is obtained by imposing the discrete symmetry

$$
\begin{array}{ll}
\mathcal{U}_{L}^{\prime}-\mathcal{U}_{L}^{\prime}, & D_{L}^{\prime}-D_{L}^{\prime}, \\
\tilde{\phi}_{1} \rightarrow \tilde{\phi}_{2}, & \tilde{\phi}_{2} \rightarrow \tilde{\phi}_{2}, \\
\mathcal{U}_{R}^{\prime}-\mathcal{U}_{R}^{\prime}, & \mathfrak{D}_{R}^{\prime} \rightarrow D_{R}^{\prime},
\end{array}
$$

where

$$
\begin{aligned}
& \psi_{L}=\frac{1}{2}\left(1-\gamma_{5}\right) \psi, \\
& \psi_{R}=\frac{1}{2}\left(1+\gamma_{5}\right) \psi .
\end{aligned}
$$

The most general Higgs-boson-quark interaction Lagrangian consistent with this discrete symmetry, and of course with $\mathrm{SU}(2) \otimes \mathrm{U}(1)$ invariance, is

$$
\begin{aligned}
\mathscr{L}_{\text {int }}= & \bar{u}_{L}^{\prime} \lambda_{1} \tilde{\phi}_{1}^{0} \mathcal{u}_{R}^{\prime}+\bar{D}_{L}^{\prime} \lambda_{1} \tilde{\phi}_{1}^{-} \bar{u}_{R}^{\prime} \\
& +\overline{\mathcal{u}}_{L}^{\prime} \lambda_{2} \tilde{\phi}_{2}^{+} D_{R}^{\prime}-\bar{D}_{L}^{\prime} \lambda_{2}\left(\tilde{\phi}_{2}^{0}\right) * D_{R}^{\prime}+\text { H.c. },
\end{aligned}
$$

where $\lambda_{1}$ and $\lambda_{2}$ are three-by-three couplingconstant matrices. From Eq. (2.2) we see that the quark mass matrices are

$$
\boldsymbol{M}_{\mathfrak{U}}^{\prime}=-\lambda_{1} \eta, \quad M_{\mathfrak{D}}^{\prime}=\lambda_{2} e^{-i \epsilon} \xi .
$$

These are diagonalized by defining mass eigenstates

$$
\begin{aligned}
& u_{L, R}=\left(\begin{array}{l}
u \\
c \\
t
\end{array}\right)_{L, R}=T_{L, R} u_{L, R}^{\prime}, \\
& D_{L, R}=\left(\begin{array}{l}
d \\
s \\
b
\end{array}\right)_{L, R}=V_{L, R} D_{L, R}^{\prime},
\end{aligned}
$$

so that

$$
\begin{aligned}
& M_{\mathcal{U}}=\left(\begin{array}{ccc}
m_{u} & 0 & 0 \\
0 & m_{c} & 0 \\
0 & 0 & m_{t}
\end{array}\right)=T_{L} M_{\mathcal{U}}^{\prime} T_{R}^{-1}, \\
& M_{\mathcal{D}}=\left(\begin{array}{ccc}
m_{d} & 0 & 0 \\
0 & m_{s} & 0 \\
0 & 0 & m_{b}
\end{array}\right)=V_{L} M_{\supset}^{\prime} V_{R}^{-1} .
\end{aligned}
$$

The combination

$$
\begin{aligned}
K & =T_{L} V_{L}^{-1} \\
& =\left(\begin{array}{ccc}
c_{1} & -s_{1} c_{3} & -s_{1} s_{3} \\
s_{1} c_{2} & c_{1} c_{2} c_{3}-s_{2} s_{3} e^{i \delta} & c_{1} c_{2} s_{3}+s_{2} c_{3} e^{i \delta} \\
s_{1} s_{2} & c_{1} s_{2} c_{3}+c_{2} s_{3} e^{i \delta} & c_{1} s_{2} s_{3}-c_{2} c_{3} e^{i \delta}
\end{array}\right)
\end{aligned}
$$

is the usual Kobayashi-Maskawa matrix ${ }^{11}$ where $s$ and $c$ refer to the sine and cosine of the respective angles. Since the lowest-order contribution 
to $K^{0}-\bar{K}^{0}$ mixing does not involve the neutral Higgs particle, we will only write down the Yukawa interactions of the charged components of $\phi_{2}$. Identifying the eigenvalues of the quark mass matrix with the quark masses and noting that

$$
M_{W}=\frac{g}{\sqrt{2}}\left(\eta^{2}+\xi^{2}\right)^{1 / 2},
$$

we find

$$
\begin{aligned}
& \mathscr{L}_{\text {int }}=\frac{g}{2 \sqrt{2} M_{W}} \phi_{2}^{+}\left\{\bar{u}\left[\frac{\xi}{\eta} M_{u} K\left(1-\gamma_{5}\right)+\frac{\eta}{\xi} K M_{\mathscr{D}}\left(1+\gamma_{5}\right)\right] \mathbb{D}\right\} \\
& + \text { H.c. , }
\end{aligned}
$$

where $M_{\mathcal{U}}$ and $M_{\mathcal{D}}$ are given by Eq. (2.12), and $K$ by Eq. (2.13).

The second model discussed in the Introduction is obtained by imposing the discrete symmetry

$$
\begin{array}{ll}
u_{L}^{\prime} \rightarrow \mathcal{u}_{L}^{\prime}, & \mathfrak{D}_{L}^{\prime} \rightarrow \mathfrak{D}_{L}^{\prime}, \\
\tilde{\phi}_{1} \rightarrow \tilde{\phi}_{1}, & \tilde{\phi}_{2} \rightarrow-\tilde{\phi}_{2}, \\
\mathcal{u}_{R}^{\prime} \rightarrow \mathcal{u}_{R}^{\prime}, & \mathfrak{D}_{R}^{\prime} \rightarrow \mathfrak{D}_{R}^{\prime} .
\end{array}
$$

This excludes Yukawa couplings of $\tilde{\phi}_{2}$ to the quarks and gives the Higgs-boson-quark interaction Lagrangian

$$
\begin{aligned}
\mathcal{L}_{\text {int }}= & \overline{\mathcal{u}}_{L}^{\prime} \lambda_{1} \tilde{\phi}_{1}^{o} u_{R}^{\prime}+\bar{D}_{L}^{\prime} \lambda_{1} \tilde{\phi}_{1}^{-} u_{R}^{\prime} \\
& +\overline{\mathcal{U}}_{L}^{\prime} \lambda_{2} \tilde{\phi}_{1}^{+} \mathfrak{D}_{R}^{\prime}-\overline{\mathfrak{D}}_{L}^{\prime} \lambda_{2}\left(\tilde{\phi}_{1}^{o}\right) * \mathfrak{D}_{R}^{\prime}+\text { H.c. }
\end{aligned}
$$

This is evaluated in exactly the same way as for model one above. The charged-Higgs-boson interaction Lagrangian is

$$
\mathscr{L}_{\text {int }}=\frac{g}{2 \sqrt{2} M_{W}} \phi_{2}^{+}\left\{\overline{\mathcal{u}}\left[\frac{\xi}{\eta} M_{\mathfrak{U}} K\left(1-\gamma_{5}\right)-\frac{\xi}{\eta} K M_{\mathfrak{D}}\left(1+\gamma_{5}\right)\right] \mathfrak{D}\right\}
$$

$$
\text { +H.c. }
$$

In both of these models, significant Higgs-boson couplings to light quarks can occur for $\xi / \eta \gg 1$. In model one, there is also the possibility $\eta / \xi \gg 1$. In this case, charged-Higgs-bos on couplings are suppressed by factors of $m_{s} / M_{W}$ and $m_{d} / M_{W}$ in light-quark processes, while on the other hand, when $\xi / \eta \gg 1$, there are Higgs-boson couplings proportional to $\left(m_{c} / M_{W}\right)$. At any rate, the $K_{L}-K_{S}$ mass difference does not provide a good upper bound for $(\eta / \xi)$. For these reasons we will focus our attention in the text on the possibility that $\xi / \eta \gg 1$. Then, the dominant contributions to $K^{0}-\bar{K}^{0}$ mixing come from the couplings which are the first terms in Eqs. (2.15) and (2.18). Thus, for the purposes of the next section, the two models are in fact identical and the bounds derived are equally valid for both models. The experimental upper limit on $D^{0}-\bar{D}^{0}$ mixing can be used to obtain an upper bound for $\eta / \xi$. This is discussed in Appendix B.
Finally, we note that charged-Higgs-boson couplings similar to Eqs. (2.15) and (2.18) will also appear in the lepton sector. If $\xi / \eta \gg 1$ (or $\eta / \xi \gg 1$ ) these can produce significant charged-Higgsboson effects involving leptons. However, even if large charged-Higgs-boson couplings occur in the quark sector, they can be virtually eliminated from the leptons by interchanging the roles of $\tilde{\phi}_{1}$ and $\widetilde{\phi}_{2}$ for leptons. Thus, significant chargedHiggs-boson couplings to quarks can be considered either with or without the analogous "large" couplings to leptons.

\section{III. $K^{0}-\bar{K}^{0}$ MIXING}

We will begin by discussing $K^{0}-\bar{K}^{0}$ mixing in the four-quark model and then extend our results to include $b$ and $t$ quarks. Thus, at first we will use the couplings of Eqs. (2.15) and (2.18) with $s_{2}=s_{3}$ $=0$. As we mentioned in the Introduction, Gaillard and Lee calculated $d^{4}$ the effect of two- $W$ boson exchange (Fig. 1) on $K^{0}-\bar{K}^{0}$ mixing. Their result is expressed in terms of an effective Hamiltonian

$$
\mathfrak{F}_{W W}=s_{1}{ }^{2} c_{1}{ }^{2} \frac{g^{4}}{2^{9} \pi^{2}} \frac{m_{c}^{2}}{M_{W}^{4}}\left[\bar{s} \gamma_{\mu}\left(1-\gamma_{5}\right) d\right]\left[\bar{s} \gamma^{\mu}\left(1-\gamma_{5}\right) d\right] .
$$

The matrix element of this effective Hamiltonian between $K^{0}$ and $\bar{K}^{0}$ states is evaluated to determine the $K^{0}-\bar{K}^{0}$ mixing from two- $W$ exchange. Since the estimated matrix element of Eq. (3.1) [ calculated by inserting the vacuum in all possible ways] is close to the experimental value for $K^{0}-\bar{K}^{0}$ mixing, ${ }^{4}$ we will require that the contributions to $K^{0}-\bar{K}^{0}$ mixing coming from chargedHiggs-boson exchange be no larger than the two$W$-boson contribution of Eq. (3.1). Since the Higgs-boson and $W$ contributions will add together we are in the end allowing the total $K^{0}-\bar{K}^{0}$ mixing to be twice as large as that given by Eq. (3.1) alone. Of course, the estimate of the matrix element of Eq. (3.1) is only approximate so our bound for $(\xi / \eta)^{2}$ will not be exact. However, since the Higgs-boson contribution of Fig. 2 is proportional to $(\xi / \eta)^{4}$ we will find that an error of a factor of $x$ in the estimate of the $K^{0}-\bar{K}^{0}$ matrix element of Eq. (3.1) will only be reflected by an error of roughly $\sqrt{x}$ in our bound. Our bound is severe enough so that such factors will not appreciably change the phenomenological implications we will discuss.

The effective Hamiltonian for $K^{0}-\bar{K}^{0}$ mixing due to Higgs-boson exchange is calculated from the diagrams of Figs. 2 and 3. As we mentioned in the previous section, we will not consider here the possibility $\eta / \xi \gg 1$ which we discuss, however, 
in Appendix B. We will neglect terms proportional to $m_{u}, m_{d}$, and $m_{s}$ relative to those proportional to $m_{c}$ (current quark masses are to be used for these parameters). The results are then identical for the two models we are considering. For the diagram of Fig. 2 we find

$$
\begin{aligned}
\mathfrak{H}_{H H}= & \frac{s_{1}^{2} c_{1}^{2}}{2}\left(\frac{g}{2 \sqrt{2} M_{W}}\right)^{4} m_{c}^{4}\left(\frac{\xi}{\eta}\right)^{2} I_{1}\left(m_{c}\right) \\
& \times\left[\bar{s} \gamma_{\mu}\left(1-\gamma_{5}\right) d\right]\left[\bar{s} \gamma^{\mu}\left(1-\gamma_{5}\right) d\right],
\end{aligned}
$$

where

$$
\begin{aligned}
I_{1}\left(m_{c}\right) & =\int_{E} \frac{d^{4} k}{(2 \pi)^{4}} \frac{k^{2}}{\left(k^{2}+m_{c}^{2}\right)^{2}\left(k^{2}+M_{H}^{2}\right)^{2}} \\
& =\frac{1}{16 \pi^{2} M_{H}{ }^{2}}+O\left(\frac{m_{c}{ }^{2}}{M_{H}^{4}}\right) .
\end{aligned}
$$

The complete expression for $I_{1}$ is given in Appen$\operatorname{dix}$ A. Thus for $M_{H}{ }^{2} \gg m_{c}^{2}$ we have

$$
\begin{aligned}
\mathfrak{F}_{H H}= & s_{1}{ }^{2} c_{1}^{2} \frac{g^{4}}{2^{11} \pi^{2}}\left(\frac{m_{c}}{M_{W}}\right)^{4} \frac{1}{M_{H}{ }^{2}}\left(\frac{\xi}{\eta}\right)^{4} \\
& \times\left[\bar{s} \gamma_{\mu}\left(1-\gamma_{5}\right) d\right]\left[\bar{s} \gamma^{\mu}\left(1-\gamma_{5}\right) d\right] .
\end{aligned}
$$

If we require that this effective Hamiltonian be no larger than the two- $W$ exchange contribution of Eq. (3.1) we obtain the bound

$$
\left(\frac{\xi}{\eta}\right)^{2} \lesssim 2\left(\frac{M_{H}}{m_{c}}\right)
$$

Of course, we must still add in the contribution from the mixed diagram of Fig. 3. However, as we will see, this will give a stricter but not very different bound than Eq. (3.5), so Eq. (3.5) is a good approximation to the actual bound derived below.

We now turn to the mixed diagram of Fig. 3. In the two models of Sec. II we find (when $\eta / \xi \ngtr>1$ )

$$
\begin{aligned}
\mathfrak{H C}_{H W}= & s_{1}{ }^{2} c_{1}{ }^{2}\left(\frac{g}{2 \sqrt{2}}\right)^{4} \frac{m_{c}{ }^{4}}{M_{W}{ }^{2}}\left(\frac{\xi}{\eta}\right)^{2}\left[4 I_{2}\left(m_{c}\right)+\frac{1}{M_{W}{ }^{2}} I_{3}\left(m_{c}\right)\right] \\
& \times\left[\bar{s} \gamma_{\mu}\left(1-\gamma_{5}\right) d\right]\left[\bar{s} \gamma^{\mu}\left(1-\gamma_{5}\right) d\right] .
\end{aligned}
$$

Expressions for $I_{2}$ and $I_{3}$ are given in Appendix A. To leading order in $1 / M_{H}{ }^{2}$ and $1 / M_{W}{ }^{2}$, the effective Hamiltonian is then

$$
\begin{aligned}
\mathfrak{F}_{H W}= & s_{1}^{2} c_{1}{ }^{2} \frac{g^{4}}{2^{11} \pi^{2}}\left(\frac{m_{c}}{M_{W}}\right)^{4} \frac{1}{M_{H}}\left(\frac{\xi}{\eta}\right)^{2}(2 A) \\
& \times\left[\bar{s} \gamma_{\mu}\left(1-\gamma_{5}\right) d\right]\left[\bar{s} \gamma^{\mu}\left(1-\gamma_{5}\right) d\right]
\end{aligned}
$$

where

$$
\begin{aligned}
A= & \frac{M_{H}{ }^{2}}{\left(M_{H}^{2}-M_{W}^{2}\right)} \ln \left(\frac{M_{H}{ }^{2}}{M_{W}^{2}}\right) \\
& -4\left[\frac{M_{W}{ }^{2}}{\left(M_{H}^{2}-M_{W}^{2}\right)} \ln \left(\frac{M_{H}{ }^{2}}{m_{c}^{2}}\right)+\frac{M_{H}^{2}}{\left(M_{W}{ }^{2}-M_{H}{ }^{2}\right)} \ln \left(\frac{M_{W}{ }^{2}}{m_{c}^{2}}\right)+1\right] .
\end{aligned}
$$

Note that $A$ is a positive quantity. Adding together Eqs. (3.4) and (3.7), we obtain the total result for charged-Higgs-boson $K^{0}-\bar{K}^{0}$ mixing in the four-quark model

$$
\begin{aligned}
\mathcal{F}_{\text {Higgs }}^{(4)} & =s_{1}{ }^{2} c_{1}{ }^{2} \frac{g^{4}}{2^{11} \pi^{2}}\left(\frac{m_{c}}{M_{W}}\right)^{4} \frac{1}{M_{H}{ }^{2}}\left[\left(\frac{\xi}{\eta}\right)^{4}+2 A\left(\frac{\xi}{\eta}\right)^{2}\right] \\
& \times\left[\bar{s} \gamma_{\mu}\left(1-\gamma_{5}\right) d\right]\left[\bar{s} \gamma^{\mu}\left(1-\gamma_{5}\right) d\right] .
\end{aligned}
$$

If we now require that the complete expression (3.9) be no larger than the $W$-exchange term of Eq. (3.1) we find the bound

$$
\left(\frac{\xi}{\eta}\right)^{2} \lesssim-A+\left(A^{2}+\frac{4 M_{H}^{2}}{m_{c}^{2}}\right)^{1 / 2}
$$

This bound is plotted as a function of $M_{H}$ for $M_{W}$ $=85 \mathrm{GeV}$ and $m_{c}=1.5 \mathrm{GeV}$ in Fig. 4. The shaded area of this plot shows the values of $(\xi / \eta)^{2}$ allowed by the bound. Also shown by the dashed line in this figure is the approximate bound of Eq. (3.5). It can be seen that the result of Eq. (3.5) is a fairly good approximation to the complete bound of Eq. (3.10).

In the six-quark model, the bounds of Eqs. (3.5) and (3.10) would still apply unless an unlikely cancellation takes place between $c$ - and $t$-quark contributions. However, the contributions of Figs. 2 and 3 with $t$ quarks in the loops are enhanced by factors of $m_{t}{ }^{4}$ so even with modest mixing angles one can obtain bounds for $(\xi / \eta)^{2}$ in the six-quark model which are even more restrictive than those of Eqs. (3.5) and (3.10). Of course, in the six-quark case our bound will involve mixing angles which are not known at present, so actual numerical values can only be given by assuming values for these mixing angles and also for the mass of the $t$ quark. The complete expression for the effective Hamiltonian coming from Figs. 2 and 3 with both $c$ and $t$ quarks in the loops is

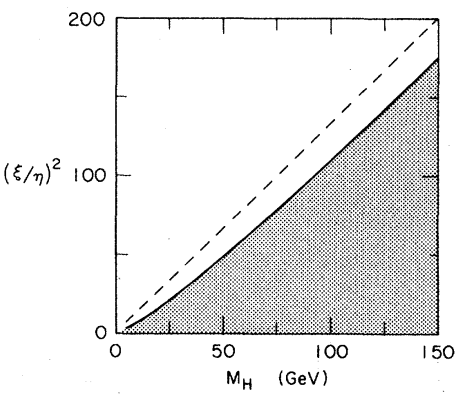

FIG. 4. The shaded region shows allowed values for $(\xi / \eta)^{2}$ in the four-quark model coming from our bound of Eq. (3.10). The dashed line shows the upper limit for $(\xi / \eta)^{2}$ given by the approximate bound of Eq. (3.5). 


$$
\begin{aligned}
\mathcal{F}_{\mathrm{Higgs}}^{(6)}=\frac{1}{2}\left(\frac{g}{2 \sqrt{2}}\right)^{4} \frac{1}{M_{W}{ }^{2}}\left(\frac{\xi}{\eta}\right)^{2}\{ & \left(c_{1} s_{2} c_{3}+c_{2} s_{3} \cos \delta\right)^{2} s_{1}{ }^{2} s_{2}{ }^{2}\left[\frac{1}{M_{W}{ }^{2}} I_{1}\left(m_{t}\right)\left(\frac{\xi}{\eta}\right)^{2}+8 I_{2}\left(m_{t}\right)+\frac{2}{M_{W}{ }^{2}} I_{3}\left(m_{t}\right)\right] m_{t}{ }^{4} \\
& +\left(c_{1} c_{2} c_{3}-s_{2} s_{3} \cos \delta\right)^{2} s_{1}{ }^{2} c_{2}{ }^{2}\left[\frac{1}{M_{W}{ }^{2}} I_{1}\left(m_{c}\right)\left(\frac{\xi}{\eta}\right)^{2}+8 I_{2}\left(m_{c}\right)+\frac{2}{M_{W}{ }^{2}} I_{3}\left(m_{c}\right)\right] m_{c}{ }^{4} \\
& \left.+2\left(c_{1} c_{2} c_{3}-s_{2} s_{3} \cos \delta\right) s_{1} s_{2} s_{1} c_{2}\left(c_{1} s_{2} c_{3}+c_{2} s_{3} \cos \delta\right)\left[\frac{1}{M_{W}{ }^{2}} I_{4}\left(\frac{\xi}{\eta}\right)^{2}+8 I_{5}+\frac{2}{M_{W}{ }^{2}} I_{6}\right] m_{t}{ }^{2} m_{c}{ }^{2}\right\} \\
\times & \times\left[\bar{s} \gamma_{\mu}\left(1-\gamma_{5}\right) d\right]\left[\bar{s} \gamma^{\mu}\left(1-\gamma_{5}\right) d\right] .
\end{aligned}
$$

The integrals $I_{1}-I_{6}$ are given in Appendix A. Since we are not concerned here with small $C P$-violating effects we have set $e^{i \delta}=\cos \delta$. Equation (3.11) is quite complicated but it has simpler forms for various values of the angles and $t$-quark mass. For example, if

$$
\begin{aligned}
\left(c_{1} s_{2} c_{3}+c_{2} s_{3} \cos \delta\right)^{2} s_{2}{ }^{2} m_{t}{ }^{4} & \\
& \ll\left(c_{1} c_{2} c_{3}-s_{2} s_{3} \cos \delta\right)^{2}{c_{2}}^{2} m_{c}{ }^{4},
\end{aligned}
$$

then the terms involving the $t$ quark are negligible and our bound is just given by the four -quark bound of Eqs. (3.5) and (3.10). If

$$
\begin{aligned}
\left(c_{1} s_{2} c_{3}+c_{2} s_{3} \cos \delta\right)^{2} s_{2}{ }^{2} m_{t}{ }^{4} & \\
& \gg\left(c_{1} c_{2} c_{3}-s_{2} s_{3} \cos \delta\right)^{2} c_{2}{ }^{2} m_{c}{ }^{4},
\end{aligned}
$$

then the term proportional to $m_{t}{ }^{4}$ will completely dominate the effective Hamiltonian. In the sixquark model two- $W$-exchange graphs also contain the heavy $t$ quark. This has been used to derive bounds on the mixing angles ${ }^{5}$ for a given $m_{t}$. When $s_{3} \ll s_{2}$, for example, those bounds have the simple form ${ }^{16} \tan ^{2} \theta_{2} \lesssim m_{c} / m_{t}$. Thus, Eq. (3.13) can be satisfied without violating these bounds on the angles coming from two- $W$ exchange.

When Eq. (3.13) is satisfied we obtain the following bound ${ }^{17}$ on $(\xi / \eta)^{2}$ :

$$
\left(\frac{\xi}{\eta}\right)^{2} \lesssim \frac{1}{I_{1}\left(m_{t}\right)}\left\{-4 M_{W}^{2} I_{2}\left(m_{t}\right)-I_{3}\left(m_{t}\right)+\left(\left[4 M_{W}{ }^{2} I_{2}\left(m_{t}\right)+I_{3}\left(m_{t}\right)\right]^{2}+\frac{I_{1}\left(m_{t}\right) m_{c}^{2}}{4 \pi^{2} a^{2} m_{t}^{4}}\right)^{1 / 2}\right\},
$$

where

$$
a^{2}=\left(c_{1} s_{2} c_{3}+c_{2} s_{3} \cos \delta\right)^{2} \frac{s_{2}{ }^{2}}{c_{1}{ }^{2}} .
$$

The results of this bound for $m_{t}=15 \mathrm{GeV}$ and $m_{t}=30 \mathrm{GeV}$ are shown in Figs. 5 and 6 , respectively. Various values of the angular factor $a$ are chosen. ${ }^{18}$ Note that the bound in the six-quark case is extremely restrictive. For quick orderof-magnitude estimates [when Eq. (3.13) is satis-

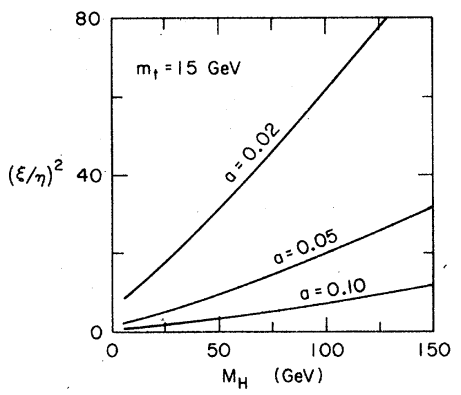

FIG. 5. The upper limits for $(\xi / \eta)^{2}$ in the six-quark model for $m_{t}=15 \mathrm{GeV}$. Various values of the angular factor $a=\left(c_{1} s_{2} c_{3}+c_{2} s_{3} \cos \delta\right) s_{2} / c_{1}$ are shown. fied] one can also use the bound

$$
\left(\frac{\xi}{\eta}\right)^{2} \lesssim \frac{2}{\sqrt{B}}\left(\frac{M_{H} m_{c}}{|a| m_{t}^{2}}\right),
$$

where

$$
B=\frac{M_{H}{ }^{2}\left(M_{H}{ }^{2}+m_{t}^{2}\right)}{\left(M_{H}^{2}-m_{t}^{2}\right)^{2}}+\frac{2 m_{t}^{2} M_{H}^{4}}{\left(M_{H}^{2}-m_{t}^{2}\right)^{3}} \ln \left(\frac{m_{t}^{2}}{M_{H}^{2}}\right) .
$$

Since the right-hand side of Eq. (3.16) is always larger than the right-hand side of Eq. (3.14) this

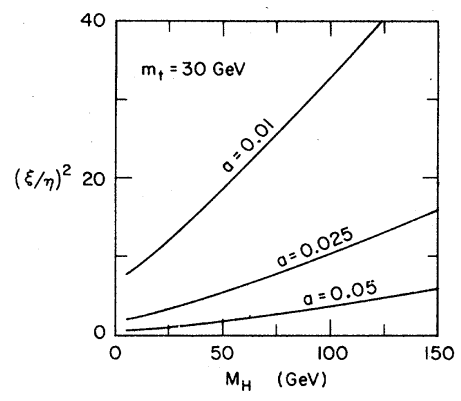

FIG. 6. Same as Fig. 5 but for $m_{t}=30 \mathrm{GeV}$. 
expression gives a valid bound for $(\xi / \eta)^{2}$ but it is not as restrictive as expression (3.14). Note that for $M_{H}^{2} \gg m_{t}^{2}, B \approx 1$.

Finally, if the parameters of the six-quark model satisfy neither condition (3.12) nor condition (3.13) the entire expression for the effective Hamiltonian (3.11) must be considered. Note that in this regime, if $\cos \delta<0$, it is possible for the last term in Eq. (3.11) to be negative ${ }^{19}$; so for certain values of the parameters a cancellation between $c$ - and $t$-quark contributions could occur. However, in most cases, the intermediate region between conditions (3.12) and (3.13) would lead to bounds more restrictive than Eq. (3.10) but not as restrictive as Eq. (3.14).

The case $\eta / \xi \gg 1$ is considered in Appendix B where a bound is derived from $D^{0}-\bar{D}^{\circ}$ mixing.

\section{STRONG-INTERACTION CORRECTIONS}

The bounds derived in the previous section were based on a consideration of the lowest-order diagrams in Figs. 2 and 3. It is the purpose of this section to discuss briefly the effects of stronginteraction corrections which we assume are described by quantum chromodynamics (QCD). Let us consider Fig. 2 in the four-quark model with massless up, down, and strange quarks. We have shown that the amplitude for the lowest-order diagram in Fig. 2 is reproduced by the tree approximation to the matrix elements of the local four-fermion operator given in Eq. (3.4). QCD corrections to this diagram can be included, to leading order in $1 / M_{H}{ }^{2}$, by using a modified effective Hamiltonian density ${ }^{20}$

$$
\begin{aligned}
\mathcal{H}_{H H}^{(\mathrm{QCD})}= & A\left(\frac{M_{H}}{\mu}, g_{s}\right) s_{1}{ }^{2}{c_{1}}^{2} \frac{g^{4}}{2^{11} \pi^{2}}\left(\frac{m_{c}}{M_{W}}\right)^{4} \frac{1}{M_{H}{ }^{2}}\left(\frac{\xi}{\eta}\right)^{4} \\
& \times\left[\bar{s} \gamma_{\mu}\left(1-\gamma_{5}\right) d\right]\left[\bar{s} \gamma^{\mu}\left(1-\gamma_{5}\right) d\right]
\end{aligned}
$$

Roughly speaking, the effect of QCD corrections will be to change the parameter $m_{c}$ in Eq. (3.4) to a running charm mass evaluated at the Higgsboson mass scale and to multiply the entire expression (3.4) by a factor coming from the anomalous dimension of the four-quark operator $\left[\bar{s} \gamma_{\mu}\left(1-\gamma_{5}\right) d\right]\left[\bar{s} \gamma^{\mu}\left(1-\gamma_{5}\right) d\right]$. This latter factor is the product of a term coming from loop momenta $p^{2}$ satisfying $M_{H}{ }^{2}<p^{2}<m_{c}^{2}$ where all four quarks are relevant, and a term coming from loop momenta smaller than $m_{c}{ }^{2}$ where only three-quark flavors act.
In the mass-independent minimal-subtraction scheme ${ }^{21} A\left(M_{H} / \mu, g_{s}\right)$ obeys the renormalizationgroup equation ${ }^{22}$

$\left[\mu \frac{\partial}{\partial \mu}+\beta\left(g_{s}\right) \frac{\partial}{\partial g_{s}}-\gamma_{+}\left(g_{s}\right)+4 \gamma_{m}\left(g_{s}\right)\right] A\left(\frac{M_{H}}{\mu}, g_{s}\right)=0$.

The quantities $\gamma_{+}\left(g_{s}\right)$ and $\gamma_{m}\left(g_{s}\right)$ are the anomalous dimensions for the operator $\left[\bar{s} \gamma_{\mu}\left(1-\gamma_{5}\right) d\right]$

$\times\left[\bar{s} \gamma^{\mu}\left(1-\gamma_{5}\right) d\right]$ and the quark mass operator, respectively. They have the perturbative expansions

$$
\begin{aligned}
& \gamma_{+}\left(g_{s}\right)=\frac{g_{s}^{2}}{4 \pi^{2}}+O\left(g_{s}^{2}\right), \\
& \gamma_{m}\left(g_{s}\right)=-\frac{g_{s}^{2}}{2 \pi^{2}}+O\left(g_{s}^{4}\right) .
\end{aligned}
$$

The $\beta$ function $\beta\left(g_{s}\right)$ has the perturbation expan$\operatorname{sion}^{23}$

$$
\beta\left(g_{s}\right)=-\left(33-2 N_{f}\right) \frac{g_{s}^{2}}{48 \pi^{2}}+O\left(g_{s}^{5}\right),
$$

where $N_{f}$ (which equals 4 here) is the number of quark flavors. Using the running coupling constant $\bar{g}_{s}\left(y, g_{s}\right)$ defined by

$$
\ln y=\int_{g_{s}}^{\bar{g}_{s}\left(y_{. g}\right)} \frac{d x}{\beta(x)}
$$

the solution to Eq. (4.2) is

$$
\begin{aligned}
A\left(\frac{M_{H}}{\mu}, g_{s}\right)= & A\left(1, \bar{g}_{s}\left(\frac{M_{H}}{\mu}, g_{s}\right)\right) \\
& \times \exp \left[-\int_{g_{s}}^{\overline{g_{s}}\left(M_{H} / \mu, g_{s}\right)} d x \frac{\gamma_{+}(x)-4 \gamma_{m}(x)}{\beta(x)}\right] .
\end{aligned}
$$

In a leading-logarithm calculation $A\left(1, \bar{g}\left(M_{H} / \mu, g_{s}\right)\right)$ may be replaced by its free-field value of 1 since $\alpha_{s}=\bar{g}_{s}^{2} / 4 \pi$ is assumed small at the mass of the Higgs boson. Using the perturbation expansions in Eqs. (4.3) and (4.4) gives

$$
A\left(\frac{M_{H}}{\mu}, g_{s}\right) \approx\left[\frac{\alpha_{s}\left(M_{H}^{2}\right)}{\alpha_{s}\left(\mu^{2}\right)}\right]^{54 / 25} \text {. }
$$

There is another large mass scale in the problem-the charm-quark mass. The matrix elements of $\left[\bar{s} \gamma_{\mu}\left(1-\gamma_{5}\right) d\right]\left[\bar{s} \gamma^{\mu}\left(1-\gamma_{5}\right) d\right]$ have a hidden dependence on the "heavy" charm-quark mass coming from loops involving virtual charmed quarks. This dependence was calculated in Ref. 6 , and in the leading-logarithmic approximation, it has the form

$$
\left\langle\left|\left[\bar{s} \gamma_{\mu}\left(1-\gamma_{5}\right) d\right]\left[\bar{s} \gamma^{\mu}\left(1-\gamma_{5}\right) d\right]\right|\right\rangle \approx\left[\frac{\alpha_{s}\left(m_{c}^{2}\right)}{\alpha_{s}\left(\mu^{2}\right)}\right]^{-6 / 25}\left[\frac{\alpha_{s}\left(m_{c}^{2}\right)}{\alpha_{s}^{\prime}\left(\mu^{2}\right)}\right]^{6 / 27}\left\langle\left|\left[\bar{s} \gamma_{\mu}\left(1-\gamma_{5}\right) d\right]\left[\bar{s} \gamma^{\mu}\left(1-\gamma_{5}\right) d\right]\right|\right\rangle^{\prime}
$$


The primed matrix elements are evaluated in an effective theory of strong interactions ${ }^{24}$ with three quark flavors $(u, d$, and $s)$, so virtual charmed quarks will no longer appear. $\alpha_{s}^{\prime}$ is the strong-coupling parameter appropriate to this theory.

Introducing a renor malization-group-invariant running charm-quark mass $\bar{m}_{c}\left(y, g_{s}, m_{c}\right)$ defined by

$$
\bar{m}_{c}\left(y, g_{s}, m_{c}\right)=m_{c} \exp \left[\int_{g_{s}}^{\bar{g}_{s}\left(y, g_{s}\right)} d x \frac{\gamma_{m}(x)}{\beta(x)}\right],
$$

so that in leading-logarithm approximation

$$
\bar{m}_{c}\left(\frac{M_{H}}{\mu}, g_{s}, m_{c}\right) \approx m_{c}\left[\frac{\alpha_{s}\left(M_{H}^{2}\right)}{\alpha_{s}\left(\mu^{2}\right)}\right]^{12 / 25}
$$

our QCD-corrected effective Hamiltonian density becomes

$$
\mathfrak{H}_{H H}^{(\mathrm{OCD})} \approx s_{1}{ }^{2} c_{1}{ }^{2} \frac{g^{4}}{2^{11} \pi^{2}}\left[\frac{\bar{m}_{c}\left(M_{H} / \mu, g_{s}, m_{c}\right)}{M_{W}}\right]^{4} \frac{1}{M_{H}{ }^{2}}\left(\frac{\xi}{\eta}\right)^{4}\left[\frac{\alpha_{s}\left(M_{H}{ }^{2}\right)}{\alpha_{s}\left(m_{c}^{2}\right)}\right]^{6 / 25}\left[\frac{\alpha_{s}\left(m_{c}{ }^{2}\right)}{\alpha_{s}^{\prime}\left(\mu^{2}\right)}\right]^{6 / 27}\left[\bar{s} \gamma_{\mu}\left(1-\gamma_{5}\right) d\right]\left[\bar{s} \gamma^{\mu}\left(1-\gamma_{5}\right) d\right] .
$$

The matrix elements of this effective Hamiltonian density are to be evaluated to all orders in the effective theory of strong interactions with three quark flavors.

Unlike the case of the two- $W$-boson exchange graph $^{6,7}$ in Fig. 1 the QCD corrections to Fig. 2 are not negligible. This happens mostly because the effective Hamiltonian density in Eq. (4.11) contains four factors of the running charm-quark mass which is small at the scale of the Higgs boson mass. The net result of this is an increase in the value of our upper bound for $(\xi / \eta)^{2}$. However, in any weak hadronic process involving Higgs-boson exchange, the QCD corrections will change the quark mass parameters in the couplings to running masses at the scale of the Higgs-boson mass. Thus a corresponding increase in $(\xi / \eta)^{2}$, from the value estimated in the absence of strong interactions, will be required to make Higgsboson exchange comparable to $W$ exchange in any weak hadronic process.

\section{CONCLUSIONS}

In this paper, we have derived bounds on charged-Higgs-boson couplings using the small experimental value for the $K_{L}-K_{S}$ mass difference. We were able to get a good bound because the diagrams in Figs. 2 and 3 , which give the chargedHiggs-boson contribution to $K^{0}-\bar{K}^{0}$ mixing, are dominated by loop momenta of order $M_{H}$ or $M_{W}$ while, due to a cancellation between the contributions of different quark flavors, Fig. 1 is dominated by loop momenta only of order $m_{c}$ or $m_{t}$.

For simplicity we have restricted our attention to the models which after spontaneous symmetry breakdown contain only a single physical chargedHiggs boson. The bounds derived can easily be applied to possible phenomenological applications of these models.

Consider, for example, the possibility of charged-Higgs-boson exchange making a significant contribution to the decay amplitude for $K$ $\rightarrow 2 \pi$. At the tree level charged-Higgs-boson and $W$-boson exchange contribute through the diagrams shown in Fig. 7. In the second model where all the couplings are proportional to $\xi / \eta$, the ratio of charged-Higgs-boson to $W$-boson exchange amplitudes arising from the tree-level diagrams in Fig. 7 is given by $Z(\xi / \eta)^{2}\left(m_{s} m_{d} / M_{H}{ }^{2}\right)$, where $Z$ is a ratio of matrix elements

$$
Z=-\frac{\left\langle 2 \pi\left|\left[\bar{s}\left(1-\gamma_{5}\right) u\right]\left[\bar{u}\left(1+\gamma_{5}\right) d\right]\right| K\right\rangle}{\left\langle 2 \pi\left|\left[\bar{s} \gamma_{\mu}\left(1-\gamma_{5}\right) u\right]\left[\bar{u} \gamma^{\mu}\left(1-\gamma_{5}\right) d\right]\right| K\right\rangle} .
$$

It has been argued ${ }^{25}$ that $Z$ could possibly be as large as about $10^{2}$. Using this and the bound in Eq. (3.5) we have that the magnitude of the ratio of charged-Higgs-boson to $W$-boson exchange is less than about $10^{2}$ times $\left(m_{s} m_{d} / M_{H} m_{c}\right)$. For reasonable current quark and Higgs-boson masses this is negligible. At the one-loop level we focus our attention on the contribution to $K \rightarrow 2 \pi$ coming from the diagrams in Fig. 8 (sometimes called "penguin" diagrams). The amplitudes arising from these diagrams are pure $\Delta I=\frac{1}{2}$, and Fig. 8 (a) has been proposed to explain the $\Delta I=\frac{1}{2}$ enhancement of weak $|\Delta S|=1$ nonleptonic decays. ${ }^{25}$ The amplitude corresponding to the Higgs-boson

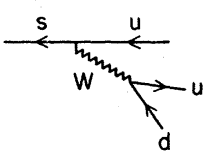

(a)

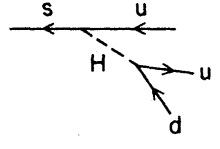

(b)
FIG. 7. Tree-level contributions to $K$ decay coming from $W$ (a) and charged-Higgs-boson (b) exchange. 


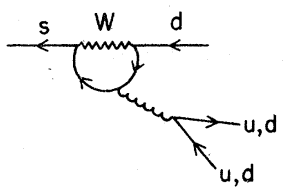

(a)

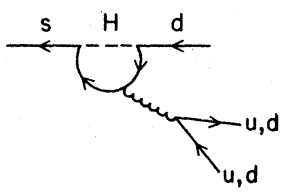

(b)
FIG. 8. "Penguin" diagrams contributing to $K$ decay through $W$ (a) and charged-Higgs-boson (b) exchange. The cycloidlike line represents a gluon.

contribution in Fig. 8(b) can easily be related to the amplitude for the $W$-boson contribution ${ }^{26}$ in Fig. 8(a) using the Fierz identity

$$
\begin{aligned}
{\left[\bar{s}\left(1+\gamma_{5}\right) c\right]\left[\bar{c}\left(1-\gamma_{5}\right) d\right] } & \\
& =-\frac{1}{2}\left[\bar{s} \gamma_{\mu}\left(1-\gamma_{5}\right) d\right]\left[\bar{c} \gamma^{\mu}\left(1+\gamma_{5}\right) c\right] .
\end{aligned}
$$

We then find that the ratio of charged-Higgsboson to $W$-boson contributions, $R$, is given by

$$
R \approx-\frac{1}{2}\left(\frac{\xi}{\eta}\right)^{2} \frac{m_{c}^{2} \ln \left(M_{H}^{2} / m_{c}^{2}\right)}{M_{H}{ }^{2} \ln \left(m_{c}^{2} / \mu^{2}\right)},
$$

where $\mu$ is a typical light hadronic mass of order $0.5 \mathrm{GeV}$. The bound of Eq. (3.5) gives

$$
|R| \lesssim\left(\frac{m_{c}}{M_{H}}\right) \frac{\ln \left(M_{H}^{2} / m_{c}^{2}\right)}{\ln \left(m_{c}^{2} / \mu^{2}\right)} .
$$

Thus for a Higgs-boson mass above $25 \mathrm{GeV}$ the $W$-boson contribution certainly dominates. A similar analysis can be performed with a $t$ quark in the loop using the six-quark bounds of Sec. III. Higher-order QCD corrections are not expected to change our conclusions since they contribute to the bound on $(\xi / \eta)^{2}$ and to the Higgs-boson contributions to the $K \rightarrow 2 \pi$ decay amplitude in roughly the same way.

As a second example we consider nonleptonic Cabibbo-allowed $D$ decays. Here we would not expect that the scalar-pseudoscalar matrix elements would be greatly enhanced over the vectoraxial-vector matrix elements. In model two (or in model one when $\eta / \xi \gg>1$ ) the ratio of Higgsboson to $W$ contributions in non-Cabibbo-suppressed $D$ decays [from diagrams analogous to Figs. $7(\mathrm{a})$ and $7(\mathrm{~b})]$ is about $\left(m_{c} m_{u} / M_{H}{ }^{2}\right)(\xi / \eta)^{2}$ and using our bound (3.5), we find that the Higgs-boson contribution can only be about $m_{u} / M_{H}$ times the $W$ contribution.

In model one, a similar analysis can be applied to the case $(\eta / \xi) \gg 1$. The present experimental upper limit for $D^{0}-\bar{D}^{0}$ mixing gives rise to the bound in Eq. (B6) of Appendix B and rules out (for a large range of Higgs-boson masses) significant Higgs-boson effects in Cabibbo-allowed nonleptonic $D$ decays, but not in $K$ decays.

Many other applications are of course possible, but we hope these simple examples are sufficient to illustrate the usefulness of the constraints on charged-Higgs-boson couplings which we have derived. While, for simplicity, we have only considered two models for the Higgs sector in this paper, it is clear that in any model (unless the Higgs-boson couplings are arranged in such a way that the leading contributions coming from the diagrams in Figs. 2 and 3 cancel,,$^{27.28}$ as in the case of the two- $W$-boson-exchange graph in Fig. $1)$, the $K_{L}-K_{S}$ mass difference will impose severe constraints on possible charged-Higgs-boson couplings. ${ }^{29}$

\section{ACKNOWLEDGMENTS}

This work was supported by the Department of Energy under Contract No. DE-AC03-76SF00515. One of us (M.B.W.) also received financial support from the National Research Council of Canada. We thank M. Barnett and G. L. Kane for stimulating discussions.

\section{APPENDIX A: USEFUL INTEGRALS}

In this appendix we tabulate the integrals used in the text, giving both exact expressions and approximations for heavy boson masses when useful:

$$
\begin{aligned}
I_{1}(m) & =\int_{E} \frac{d^{4} k}{(2 \pi)^{4}}\left[\frac{k^{2}}{\left(k^{2}+m\right)^{2}\left(k^{2}+M_{H}{ }^{2}\right)^{2}}\right] \\
& =\frac{1}{16 \pi^{2}}\left[\frac{M_{H}{ }^{2}+m^{2}}{\left(M_{H}{ }^{2}-m^{2}\right)^{2}}+\frac{2 m^{2} M_{H}^{2}}{\left(M_{H}{ }^{2}-m^{2}\right)^{3}} \ln \left(\frac{m^{2}}{M_{H}{ }^{2}}\right)\right]=\frac{1}{16 \pi^{2} M_{H}{ }^{2}}+O\left(\frac{m^{2}}{M_{H}{ }^{4}}\right), \\
I_{2}(m) & =\int_{E} \frac{d^{4} k}{(2 \pi)^{4}}\left[\frac{1}{\left(k^{2}+m^{2}\right)^{2}\left(k^{2}+M_{H}{ }^{2}\right)\left(k^{2}+M_{W}{ }^{2}\right)}\right] \\
& =\frac{1}{16 \pi^{2}}\left[\frac{M_{H}{ }^{2} \ln \left(m^{2} / M_{H}{ }^{2}\right)}{\left(M_{H}{ }^{2}-m^{2}\right)^{2}\left(M_{H}{ }^{2}-M_{W}{ }^{2}\right)}+\frac{M_{W}{ }^{2} \ln \left(m^{2} / M_{W}{ }^{2}\right)}{\left(M_{W}{ }^{2}-m^{2}\right)^{2}\left(M_{W}{ }^{2}-M_{H}{ }^{2}\right)}-\frac{1}{\left(m^{2}-M_{H}{ }^{2}\right)\left(m^{2}-M_{W}{ }^{2}\right)}\right] \\
& =\frac{-1}{16 \pi^{2} M_{W}{ }^{2} M_{H}{ }^{2}}\left[\frac{M_{W}{ }^{2} \ln \left(M_{H}{ }^{2} / m^{2}\right)}{\left(M_{H}{ }^{2}-M_{W}{ }^{2}\right)}+\frac{M_{H}{ }^{2} \ln \left(M_{W}{ }^{2} / m^{2}\right)}{\left(M_{W}{ }^{2}-M_{H}{ }^{2}\right)}+1\right]\left[1+O\left(\frac{m^{2}}{M_{H}{ }^{2}}, \frac{m^{2}}{M_{W}{ }^{2}}\right)\right],
\end{aligned}
$$




$$
\begin{aligned}
& I_{3}(m)=\int_{E} \frac{d^{4} k}{(2 \pi)^{4}}\left[\frac{k^{2}}{\left(k^{2}+m^{2}\right)^{2}\left(k^{2}+M_{H}^{2}\right)\left(k^{2}+M_{W}^{2}\right)}\right] \\
& =\frac{1}{16 \pi^{2}}\left[\frac{M_{H}{ }^{4} \ln \left(M_{H}{ }^{2} / m^{2}\right)}{\left(M_{H}{ }^{2}-M_{W}{ }^{2}\right)\left(m^{2}-M_{H}{ }^{2}\right)^{2}}+\frac{M_{W}{ }^{4} \ln \left(M_{W}{ }^{2} / m^{2}\right)}{\left(M_{W}{ }^{2}-M_{H}{ }^{2}\right)\left(m^{2}-M_{W}{ }^{2}\right)^{2}}+\frac{m^{2}}{\left(m^{2}-M_{H}{ }^{2}\right)\left(m^{2}-M_{W}{ }^{2}\right)}\right] \\
& =\frac{1}{16 \pi^{2}}\left[\frac{\ln \left(M_{H}{ }^{2} / M_{W}{ }^{2}\right)}{\left(M_{H}{ }^{2}-M_{W}{ }^{2}\right)}\right]\left[1+O\left(\frac{m^{2}}{M_{H}{ }^{2}}, \frac{m^{2}}{M_{W}{ }^{2}}\right)\right] \text {, } \\
& I_{4}=\int_{E} \frac{d^{4} k}{(2 \pi)^{4}}\left[\frac{k^{2}}{\left(k^{2}+m_{c}^{2}\right)\left(k^{2}+m_{t}^{2}\right)\left(k^{2}+M_{H}^{2}\right)^{2}}\right]
\end{aligned}
$$

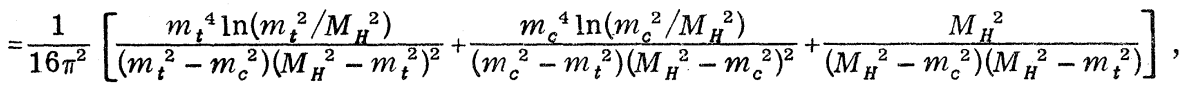

$$
\begin{aligned}
& I_{5}=\int_{E} \frac{d^{4} k}{(2 \pi)^{4}}\left[\frac{1}{\left(k^{2}+m_{c}{ }^{2}\right)\left(k^{2}+m_{t}{ }^{2}\right)\left(k^{2}+M_{H}{ }^{2}\right)\left(k^{2}+M_{W}{ }^{2}\right)}\right] \\
& =\frac{1}{16 \pi^{2}}\left[\frac{M_{H}{ }^{2} \ln \left(M_{W}{ }^{2} / M_{H}{ }^{2}\right)}{\left(M_{H}{ }^{2}-M_{W}{ }^{2}\right)\left(M_{H}{ }^{2}-m_{t}{ }^{2}\right)\left(M_{H}{ }^{2}-m_{c}{ }^{2}\right)}+\frac{m_{t}{ }^{2} \ln \left(M_{W}{ }^{2} / m_{t}{ }^{2}\right)}{\left(m_{t}{ }^{2}-M_{W}{ }^{2}\right)\left(m_{t}{ }^{2}-M_{H}{ }^{2}\right)\left(m_{t}{ }^{2}-m_{c}{ }^{2}\right)}\right. \\
& \left.+\frac{m_{c}^{2} \ln \left(M_{W}^{2} / m_{c}^{2}\right)}{\left(m_{c}^{2}-M_{W}^{2}\right)\left(m_{c}^{2}-M_{H}^{2}\right)\left(m_{c}^{2}-m_{t}^{2}\right)}\right] \text {, } \\
& I_{6}=\int_{E} \frac{d^{4} k}{(2 \pi)^{4}}\left[\frac{k^{2}}{\left(k^{2}+m_{c}^{2}\right)\left(k^{2}+m_{t}^{2}\right)\left(k^{2}+M_{H}^{2}\right)\left(k^{2}+M_{W}^{2}\right)}\right] \\
& =\frac{1}{16 \pi^{2}}\left[\frac{M_{H}^{4} \ln \left(M_{H}{ }^{2} / M_{W}{ }^{2}\right)}{\left(M_{H}{ }^{2}-M_{W}{ }^{2}\right)\left(M_{H}{ }^{2}-m_{t}^{2}\right)\left(M_{H}{ }^{2}-m_{c}^{2}\right)}+\frac{m_{t}^{4} \ln \left(m_{t}^{2} / M_{W}{ }^{2}\right)}{\left(m_{t}^{2}-M_{W}^{2}\right)\left(m_{t}^{2}-M_{H}^{2}\right)\left(m_{t}^{2}-m_{c}^{2}\right)}\right. \\
& \left.+\frac{m_{c}^{4} \ln \left(m_{c}^{2} / M_{W}^{2}\right)}{\left(m_{c}^{2}-M_{W}^{2}\right)\left(m_{c}^{2}-M_{H}^{2}\right)\left(m_{c}^{2}-m_{t}^{2}\right)}\right] \text {. }
\end{aligned}
$$

\section{APPENDIX B : $D^{0}-\bar{D}^{0}$ MIXING}

In model one there is a possibility of significant charged-Higgs-boson couplings when $(\eta / \xi) \gg 1$. The $K^{0}-\bar{K}^{0}$ mass difference does not provide a strict bound on $(\eta / \xi)^{2}$ since, for $(\eta / \xi) \gg 1$, the leading contribution cancels out from the Higgs boson-exchange graphs in Figs. 2 and 3 when the amplitudes corresponding to different quarks in the loop are added together. A better bound can be achieved by studying $D^{0}-\bar{D}^{0}$ mixing. We begin by considering the four-quark model where $s_{2}$ $=s_{3}=0$.

Experimentally ${ }^{1}$

$$
\frac{\Gamma\left(D^{0} \rightarrow \bar{D}^{0} \rightarrow K^{+} \pi^{-}\right)}{\Gamma\left(D^{0} \rightarrow K \pi\right)} \leqslant 0.16,
$$

which implies that ${ }^{30}$

$$
\Delta m_{D} \leqslant 0.6 \Gamma_{D} \text {. }
$$

Estimating the total width for $D$ decay by assuming that the charm quark decays to free particles that dress themselves into physical states with unit probability gives

$$
\Gamma_{D} \simeq \frac{5 G^{2} m_{c}^{5}}{192 \pi^{3}} \simeq 8.6 \times 10^{-13} \mathrm{GeV} .
$$

In the case $(\eta / \xi) \gg 1$, the two-charged-Higgs- boson exchange graph will dominate over the graph with one Higgs boson and one $W$ boson exchanged. The usual box diagram with two $W$ bosons in the loop also contributes to $D^{\circ}-\bar{D}^{\circ}$ mixing. However, since in this case the loop is dominated by small momenta of order the strange-quark mass, there is no justification for a local effective Hamiltonian formulation. On the other hand, the two-Higgs boson-exchange diagram is dominated by large loop momenta of order the Higgs mass and gives rise to an effective Hamiltonian density

$$
\begin{aligned}
\mathfrak{F}_{H H} & =s_{1}{ }^{2} c_{1}{ }^{2} \frac{g^{4}}{2^{11} \pi^{2}}\left(\frac{m_{s}}{M_{W}}\right)^{4} \frac{1}{M_{H}^{2}}\left(\frac{\eta}{\xi}\right)^{4} \\
& \times\left[\bar{c} \gamma_{\mu}\left(1+\gamma_{5}\right) u\right]\left[\bar{c} \gamma^{\mu}\left(1+\gamma_{5}\right) u\right]\left[1+O\left(\frac{m_{S}^{2}}{M_{H}^{2}}\right)\right] .
\end{aligned}
$$

The contribution to the $D_{L}-D_{S}$ mass difference given by the matrix elements of the effective Hamiltonian in Eq. (B4), evaluated by inserting the vacuum in all possible ways, is

$$
\Delta m_{D} \approx \frac{1}{4}\left(\frac{\eta}{\xi}\right)^{4} \frac{m_{s}^{2}}{M_{H}^{2}} \times 10^{-16} \mathrm{GeV} .
$$

Requiring that this contribution be no longer than 
the experimental upper limit gives the bound

$$
\left(\frac{\eta}{\xi}\right)^{2} \lesssim 10^{2}\left(\frac{M_{H}}{m_{s}}\right)
$$

In the six-quark model the box diagrams with a virtual $b$ quark in the loop will also contribute to $D^{0}-\bar{D}^{0}$ mixing. Then bounds on $(\eta / \xi)^{2}$ can be derived for a given value of the mixing angles much as was done in the latter part of Sec. III where $K^{0}-\bar{K}^{0}$ mixing was discussed.
${ }^{1}$ Particle Data Group, Phys. Lett. 75B, 1 (1978). ${ }^{2}$ S. L. Glashow, J. Iliopoulos, and L. Maiani, Phys. Rev. D 2, 1285 (1970).

${ }^{3}$ S. Weinberg, Phys. Rev. Lett. 19, 1264 (1967); A. Salam, in Elementary Particle Theory: Relativistic Groups and Analyticity (Nobel Symposium No. 8), edited by N. Svartholm (Almqvist and Wiksell, Stockholm, 1968), p. 367.

${ }^{4}$ M. K. Gaillard and B. W. Lee, Phys. Rev. D $\underline{10}, 897$ (1974).

${ }^{5}$ J. Ellis, M. K. Gaillard, D. V. Nanopoulos, and S. Rudaz, Nucl. Phys. B131, 285 (1977); V. Barger, W. F. Long, and S. Pakvasa, Phys. Rev. Lett. 42 , 1585 (1979); R. E. Shrock, S. B. Treiman, and LingLie Wang, ibid. 42, 1589 (1979); L. Wolfenstein, Carnegie-Mellon University Report No. COO-3066, 1979 (unpublished).

${ }^{6}$ E. Witten, Nucl. Phys. B122, 109 (1977).

${ }^{7}$ A. I. Vainshtein, V. I. Zakharov, V. A. Novikov, and M. A. Shifman, Yad. Fiz. 23, 1024 (1976) [Sov. J. Nucl. Phys. 23, 540 (1976)].

${ }^{8} \mathrm{~A}$ property is called natural if it is true for arbitrary values of the parameters in the Lagrangian.

${ }^{9}$ S. L. Glashow and S. Weinberg, Phys. Rev. D 15,1958 (1977).

${ }^{10}$ S. Weinberg, Phys. Rev. Lett. 37, 657 (1976).

${ }^{11}$ M. Kobayashi and T. Maskawa, Prog. Theor. Phys. 49, 652 (1973).

${ }^{12}$ H. E. Haber, G L. Kane, and T. Sterling, University of Michigan Report No. UM-HE 78-45, 1978 (unpublished).

${ }^{13}$ P. Sikivie, Phys. Lett. 65B, 141 (1976); N. G. Deshpande and E. Ma, Phys. Rev. D 18, 2574 (1978).

${ }^{14} \mathrm{H}$. Georgi and D. V. Nanopoulos, Phys. Lett. $82 \mathrm{~B}, 95$ (1979).

${ }^{15}$ This was verified by explicit calculation in Ref. 13.

${ }^{16}$ H. Harari, Phys. Rep. 42C, 235, (1978).

${ }^{17}$ Since the matrix elements of the effective Hamiltonian density in Eq. (3.1) (calculated by inserting the vacuum in all possible ways) come close to saturating the experimental value for $K^{0}-\bar{K}^{0}$ mixing, we derive our bound by demanding that the matrix elements of the effective Hamiltonian in Eq. (3.11) be no larger than those of the effective Hamiltonian in Eq. (3.1).

${ }^{18} \mathrm{Smaller}$ values of $a$ are used for the larger value of $m_{t}$ so that the simple bound $\tan ^{2} \theta_{2} \lesssim m_{c} / m_{t}$ is not violated.

${ }^{19}$ We adopt the following phase convention [see F. Gilman and M. Wise, Phys. Lett. 83B, 83 (1979)]. All the angles $\theta_{j}, j \in\{1,2,3\}$, are chosen to lie in the first quadrant so their sines and cosines are positive.

${ }^{20}$ The power counting required to justify a local effective Hamiltonian formulation to all orders in the strong coupling was done in sec. II of Ref. 6 .

${ }^{21}$ S. Weinberg, Phys. Rev. D 8, 3497 (1973); G. 't Hooft, Nucl. Phys. B61, 455 (1973).

${ }^{22}$ M. Gell-Mann and F. Low, Phys. Rev. 95, 1300 (1954); E. C. G. Stuekelberg and A. Peterman, Helv. Phys. Acta 26, 499 (1953); G. C. Gallan, Phys. Rev. D $\underline{2}$, 1541 (1970); K. Symanzik, Commun. Math. Phys. 18, 227 (1970).

${ }^{23}$ D. J. Gross and F. Wilczek, Phys. Rev. Lett. 30, 1343 (1973); H. D. Politzer, ibid. 30, 1346 (1973).

${ }^{24} \mathrm{~A}$ detailed discussion of what is meant by an effective theory of strong interactions can be found in Sec. III of E. Witten, Nucl. Phys. B104, 445 (1976).

${ }^{25}$ A. I. Vainshtein, V. I. Zakharov, and M. A. Shifman, Zh. Eksp. Teor. Fiz. Pis'ma Red.; 22, 123 (1975) [JETP Lett. 22, 55 (1975)]; M. A. Shifman, A. I. Vainshtein, and V. I. Zakharov, Nucl. Phys. B120, 316 (1977).

${ }^{26} \mathrm{M}$. Wise and E. Witten, [Phys. Rev. D 20, 1216 (1979)] have shown how the local four-fermion structure of the effective Hamiltonian arising from a calculation of the one-loop diagram in Fig. 8(a) is preserved, despite the presence of multiple soft-gluon exchange in higher orders.

${ }^{27} \mathrm{~A}$ model that falls into this class has been proposed in G. L. Kane, SLAC Report No. SLAC-PUB-2326, 1979 (unpublished).

${ }^{28}$ The implications of $K^{0}-\bar{K}^{0}$ mixing for models in this class has been discussed in J. A. Grifols, Phys. Rev. D 18, 2704 (1978).

${ }^{29}$ After completion of this work it was brought to our attention that similar conclusions have been reached by C. T. Hill in his Ph. D. thesis (unpublished). ${ }^{30}$ See, for example, J. J. Sakurai, Invariance Principles and Elementary Particles (Princeton Univ. Press, Princeton, New Jersey, 1964). 